\title{
On the Dynamics of Influence Networks via Reflected Appraisal
}

\author{
Peng Jia, Anahita Mirtabatabaei, Noah E. Friedkin, Francesco Bullo
}

\begin{abstract}
In any modern society, individuals interact to form opinions on various topics, including economic, political, and social aspects. Opinions evolve as the result of the continuous exchange of information among individuals where interpersonal influences play the key role. The study of influence network evolution has wide applications in the field of social organization and social psychology. A compelling model is Friedkin's reflected appraisal model where each individual's self-appraisal is set equal to the relative control and power that the agent exerted over prior issue outcomes. Motivated by this empirical framework, we (i) present a rigorous mathematical formulation of the reflected appraisal influence network dynamics, (ii) study the equilibria and the convergence properties of the dynamical influence systems, and (iii) construct the social conditions leading to the emergence of single opinion leaders, clusters of leaders, or diffuse and democratic power structures. In particular, an appropriatelydefined eigenvector centrality of the influence network is proved to determine each individual's social power and self-appraisal evolution, and then determine the opinion formulation of the whole network.
\end{abstract}

\section{INTRODUCTION}

This paper advances the analysis of Friedkin's (2011) dynamical model of the evolution of power along opinion formation processes in interpersonal influence networks. Specifically, we study the mathematics of the sociological and psychological mechanism of reflected appraisal and its effects on sequential opinion formation processes.

Classic references on influence networks and opinion formation processes in sociology include (French, 1956; Harary, 1959; DeGroot, 1974; Friedkin and Johnsen, 1999), and more recent works in the multi-agent systems literature include (Deffuant et al., 2000; Hegselmann and Krause, 2002; Acemoglu et al., 2013; Blondel et al., 2009). Opinion formation processes are classically described by averaging algorithms and are closely related to the burgeoning literature on consensus algorithms.

The assumption of a static influence network for a particular issue is widely adopted. Starting from this assumption, the model in (Friedkin, 2011) considers an evolution process of the influence network across issues, over time, as the group moves from issue to issue in a lengthy sequence of issues. The evolution process is motivated by the sociological literature on reflected appraisals (Cooley, 1902; Gecas and Schwalbe, 1983), which finds that individuals' self-appraisals on some dimension (e.g., self-confidence, self-esteem, self-worth) are

This work was supported by the UCSB Institute for Collaborative Biotechnology through grant W911NF-09-D-0001 from the U.S. Army Research Office. The content of the information does not necessarily reflect the position or the policy of the Government, and no official endorsement should be inferred.

The authors are affiliated with the Center for Control, Dynamical Systems and Computation, University of California at Santa Barbara, \{pjia,mirtabatabaei, bullo\}@engineering.ucsb.edu. Noah Friedkin is affiliated with the Department of Sociology, University of California at Santa Barbara, friedkin@soc.ucsb.edu. influenced by the appraisals of other individuals of them. In a social influence network, the available evidence (Friedkin, 2011) indicates that this mechanism links individuals' selfweights, i.e., the extent to which they are open or closed to interpersonal influence on an issue, with their relative control over past issue outcomes. In turn, these evolving self-weights have important effects on the distribution of relative control over future issue outcomes. In other words, we study a model of network evolution, in which opinion formation processes are affected by, and in turn affect, the influence network.

In this paper, we move from the simulation results in (Friedkin, 2011) to the mathematical analysis of the evolution process for the influence network. The analysis is simplified by a scope restriction to the classic (DeGroot, 1974) model of opinion dynamics on a particular issue. This classic model is a special case of the (Friedkin and Johnsen, 1999) model upon which (Friedkin, 2011) is based. In the DeGroot model, (i) an influence network is precisely described by a single averaging matrix, (ii) opinion consensus is known to arise if the influence network is strongly connected (and satisfies a weak aperiodicity assumption), (iii) each agent is known to have a strictly positive influence on the final opinion outcome. In this paper we analyze the reflected appraisal mechanism (Friedkin, 2011) for the evolution of social power and self-appraisal for the special case in which the opinion formation process is described by the DeGroot model achieving opinion consensus on each of a sequence of issues.

Contributions. We consider the above-described network evolution process, which for brevity we refer to as the DeGrootFriedkin model. As the first contribution, we provide an explicit and concise mathematical formulation of the reflected appraisal mechanism for network evolution as a discrete-time nonlinear system defined over a simplex. As a second set of contributions, we study the equilibria and the asymptotic convergence properties of this nonlinear system. We provide a complete mathematical analysis of two special cases and an incomplete set of mathematical results, complemented by statistical evaluations, for the general case.

The combined results of the mathematical and statistical analysis are summarized as follows. For all influence networks described by an appropriately-defined eigenvector centrality score $c$, there exists a unique value of self-weights $x^{*}(c)$ that is invariant and unchanged under the reflected appraisal process (a fixed point of the corresponding dynamical system). Moreover, trajectories of the network evolution process converge to this fixed point $x^{*}(c)$ from almost all initial conditions (the only exception being autocratic configurations in which all agents have vanishing self-weights, except for the autocrat agent). Finally, this equilibrium self-weight configuration $x^{*}(c)$ has the following three properties. The 
first property is: if the eigenvector centrality scores $c$ are uniform, then the self-weights $x^{*}(c)$ are uniform (a notion of democracy). The second property is: if the eigenvector centrality scores $c$ are maximally non-uniform in the sense that the influence topology is a star, then the self-weights $x^{*}(c)$ are maximally non-uniform in an autocratic configuration (where the autocrat agent is the center node of the star topology). The third property is: if the eigenvector centrality scores $c$ represent an intermediate regime, then the equilibrium selfweights $x^{*}(c)$ has the same "rank order" as $c$, in the sense that if a node $i$ has a larger centrality score than $j$, then their corresponding equilibrium self-weights will be have the same relationship. These results are related to the discussions in (Friedkin, 2011), where the reflected mechanism is paired with the more general (Friedkin and Johnsen, 1999) opinion formation model.

We illustrate our results with applications to Krackhardt's advice network (Krackhardt, 1987). Due to space limitations, we postpone complete proofs for several statements to further submissions.

Notation. In the Euclidean space $\mathbb{R}^{n}$, we define $\mathbb{1}_{n}=$ $(1, \ldots, 1)^{T}$ and $\mathbb{O}_{n}=(0, \ldots, 0)^{T}$. Moreover, for $i \in$ $\{1, \ldots, n\}$, we let $\mathbb{e}_{i}$ be the vector with all entries equal to 0 except for the $i$ th entry equal to 1 . Given $\left(x_{1}, \ldots, x_{n}\right) \in \mathbb{R}^{n}$, let $\operatorname{diag}(x)$ denote the diagonal $n \times n$ matrix whose diagonal entries are $x_{1}, \ldots, x_{n}$. We also recall the definition of the $n$ simplex $\Delta_{n}=\left\{x \in \mathbb{R}^{n} \mid x \geq 0, \mathbb{1}_{n}^{T} x=1\right\}$.

\section{THE DEGROOT-FrIEDKIN MODEL}

The dynamical model of self-weight is based upon two basic ideas: First, we adopt the DeGroot model for the dynamics of opinion over a single issue. Second, we adopt the Friedkin model for the dynamics of self-weight and power over a sequence of issues. Specifically, the model is defined as follows.

\section{A. State variables}

We consider a group of $n \geq 2$ agents. Each agent $i \in$ $\{1, \ldots, n\}$ has an initial opinion $y_{i}(s) \in \mathbb{R}$ about a sequence of issues $s \in \mathbb{N}$ and a measure of self-weight $x(s) \in \Delta_{n}$ that evolves as the issues are discussed.

\section{B. Dynamics of opinions about an issue}

At fixed issue $s$ and fixed self-weight vector $x(s)$, the vector of opinions about issue $s$ is a trajectory $t \mapsto y(s, t) \in \mathbb{R}^{n}$ that evolves according to the DeGroot averaging model:

$$
y(s, t+1)=W(x(s)) y(s, t),
$$

with initial conditions $y(s, 0)=y(s)$ and with $n \times n$ influence matrix $W(x)$ defined as follows.

We assume the agents average their opinions with weights that incorporate two contributions: the agents' self-weights $x$ and a given fixed network of interpersonal interactions. Specifically, we introduce a relative interaction matrix matrix $C$ describing a network of relative interpersonal accorded weights: the $n \times n$ matrix $C$ is assumed to be non-negative, row-stochastic and to have zero diagonal. In summary, following (Friedkin, 2011), the $n \times n$ influence matrix $W(x)$, for $x \in \Delta_{n}$, is defined by

$$
\begin{aligned}
W(x) & =\operatorname{diag}(x)+\left(I_{n}-\operatorname{diag}(x)\right) C \\
& =\operatorname{diag}(x) I_{n}+\operatorname{diag}\left(\mathbb{1}_{n}-x\right) C,
\end{aligned}
$$

where the diagonal matrix $\operatorname{diag}(x)$ measures the relative contribution of the self-weights and the relative interaction matrix $C$, scaled by the diagonal matrix $\left(I_{n}-\operatorname{diag}(x)\right)$, measures the relative contribution of the network interactions.

Lemma 1 (Properties of the influence matrix) Given a self-weight vector $x \in \Delta_{n}$ and a relative interaction (rowstochastic and with zero diagonal) matrix $C \in \mathbb{R}^{n \times n}$ that is irreducible, the following statements hold:

(i) the influence matrix $W(x)$, as defined in the decomposition (2), is non-negative and row stochastic,

(ii) for all $x \in \Delta_{n}$, there exists a unique vector $w(x) \in \Delta_{n}$ such that $w(x)^{T} W(x)=w(x)^{T}$ and $\lim _{t \rightarrow \infty} W(x)^{t}=$ $\mathbb{1}_{n} w(x)^{T}$,

(iii) if $x \in \Delta_{n} \backslash\left\{\mathbb{e}_{1}, \ldots, \mathbb{e}_{n}\right\}$, then $W(x)$ is irreducible, the digraph associated to $W(x)$ is strongly connected, and $w(x)>0$, and

(iv) if $x=\mathbb{e}_{i}$ for some $i \in\{1, \ldots, n\}$, then $W\left(\mathbb{e}_{i}\right)$ is reducible, the node $i$ is the only globally reachable node in the digraph associated to $W\left(\mathbb{e}_{i}\right)$, and $w(x)=\mathbb{e}_{i}$.

We refer to the vector $w(x) \in \Delta_{n}$ as the dominant left eigenvector of $W(x)$.

Lemma 1 leads to the well-understood consensus outcome of the opinion formation process (about issue $s$ ):

$$
\lim _{t \rightarrow \infty} y(s, t)=\left(\lim _{t \rightarrow \infty} W(x(s))^{t}\right) y(s)=\left(w(x(s))^{T} y(s)\right) \mathbb{1}_{n} .
$$

In other words, the agents' opinions converge to a consensus value $w(x(s))^{T} y(s)$, which is a convex combination of their initial opinions $y(s)$ with convex combination coefficients $w(x(s))$ describing the relative contributions of each agent.

Remark 2 (Reducible relative interactions with globally reachable nodes) It is possible to generalize Lemma 1 to the setting of reducible relative interaction matrices $C$. Let $G(C)$ be the digraph associated to $C$ by assuming that $(i, j)$ is an edge if and only if $C_{i j}>0$ or, equivalently, by requiring the digraph $G(C)$ to have adjacency matrix $C$. Then $C$ is reducible if and only if $G(C)$ is not strongly connected. Now, assume that $G(C)$ contains at least one globally reachable node and, without loss of generality, assume the globally reachable nodes are $\{1, \ldots, m\}$, for $m \leq n$. If $x \in \Delta_{n} \backslash\left\{\mathbb{e}_{m+1}, \ldots, \mathbb{e}_{n}\right\}$, then there exists a unique $w(x) \in \Delta_{n}$ such that $w(x)^{T} W(x)=$ $w(x)^{T}, w_{m+1}(x)=\cdots=w_{n}(x)=0$, and $\lim _{t \rightarrow \infty} W(x)^{t}=$ $\mathbb{1}_{n} w(x)^{T}$. Moreover, $\mathbb{e}_{i}^{T} W\left(\mathbb{e}_{i}\right)=\mathbb{e}_{i}^{T}$ for $i \in\{1, \ldots, m\}$.

In other words, agents who are not globally reachable in $G(C)$ have no influence in the final opinion value. In what follows we will focus on irreducible matrices, with the understanding that the role of un-influential agents is simple to include and analyze. 


\section{Dynamics of self-weights across a sequence of issues}

We assume the relative interaction matrix $C$ is irreducible so that the influence matrix $W(x)$ has a unique dominant left eigenvector for all $x \in \Delta_{n}$.

As a function of a sequence of issues $s \in \mathbb{N}$, the selfweight vector $\{x(s)\}_{s \in \mathbb{N}}$ evolves as follows: $x(1) \in \Delta_{n}$ is an arbitrary initial condition, and $x(s+1)$ is defined to be the dominant left eigenvector $w(x(s))$ of the influence matrix $W(x(s))$, normalized so that $\mathbb{1}_{n}^{T} w(x(s))=1$. We refer to this dynamical evolution of the self-appraisal scores $x$ as the DeGroot-Friedkin model.

According to this reflected appraisal mechanism, the selfweight of an agent is set equal to the relative control and power that the agent exerted over prior issue outcomes. Quoting from Friedkin (2011), “The empirical findings of the experiment support a reflected appraisal effect in which individuals dampen or elevate their self-weights, becoming more or less open to interpersonal influence, in correspondence with their prior relative control over group issue outcomes across a sequence of issues."

\section{Problem statement}

We are interested in characterizing the existence and stability of equilibria for the DeGroot-Friedkin model as a function of the network structure and parameters as embodied in the relative interaction matrix $C$. Moreover, we are interested in the properties of certain specific self-weight vectors: we define the equal-weight vector $\mathbb{1}_{n} / n$ and the single-leader vectors $\left\{\mathbb{e}_{1}, \ldots, \mathbb{e}_{n}\right\}$.

\section{E. Explicit mathematical modelling}

Given a relative interaction matrix $C$ (row-stochastic with zero diagonal) that is irreducible, let $c \in \Delta_{n}$ be the unique positive left eigenvector of $C$ associated with the eigenvalue 1 and normalized so that $\mathbb{1}_{n}^{T} c=1$. As in the proof of Lemma 1, the existence of the $c$ follows from the Perron-Frobenius Theorem. As in Lemma 1, we refer to $c$ as the dominant left eigenvector of $C$. (We adopt this nomenclature for simplicity, even if it is a slight abuse of notation for matrices $C$ that are not necessarily primitive.)

Lemma 3 (Self-weight dynamics with centrality scores) For $n \geq 2$, assume the matrix $C \in \mathbb{R}^{n \times n}$ is row stochastic, irreducible and has zero diagonal and let the positive $c \in \Delta_{n}$ be its dominant left eigenvector. The DeGroot-Friedkin model is equivalent to $x(s+1)=F(x(s))$, where the continuous map $F: \Delta_{n} \rightarrow \Delta_{n}$ is defined by

$F(x)=\left\{\begin{array}{l}\mathbb{e}_{i}, \quad \text { if } x=\mathbb{e}_{i} \text { for any } i \in\{1, \ldots, n\}, \\ \left(\frac{c_{1}}{1-x_{1}}, \ldots, \frac{c_{n}}{1-x_{n}}\right) / \sum_{i=1}^{n} \frac{c_{i}}{1-x_{i}}, \quad \text { otherwise. }\end{array}\right.$

Sketch of proof: Given the self-weight $x(s) \in \Delta_{n}$ at issue $s$, the subsequent self-weight vector is defined by
$W(x(s))^{T} x(s+1)=x(s+1)$ and $x(s+1) \in \Delta_{n}$. We are therefore interested in the equality

$$
\left(\operatorname{diag}(x(s))+\left(I_{n}-\operatorname{diag}(x(s))\right) C\right)^{T} x(s+1)=x(s+1) .
$$

This equality leads to

$$
\operatorname{diag}\left(\mathbb{1}_{n}-x(s)\right) x(s+1)=C^{T} \operatorname{diag}\left(\mathbb{1}_{n}-x(s)\right) x(s+1),
$$

which implies that the vector $\operatorname{diag}\left(\mathbb{1}_{n}-x(s)\right) x(s+1)$ is a left eigenvector of $C$ associated with eigenvalue 1 . Therefore, $x(s+1) \in \Delta_{n}$ satisfies $\operatorname{diag}\left(\mathbb{1}_{n}-x(s)\right) x(s+1)=\alpha(s) c$, where the scaling coefficient $\alpha(s)>0$ is computed so that $\mathbb{1}_{n}^{T} x(s+1)=1$. In other words, we have $\left(1-x_{j}(s)\right) x_{j}(s+$ $1)=\alpha(s) c_{j}$ for all $j \in\{1, \ldots, n\}$. If $x(s)=\mathbb{e}_{i}$ for some $i \in\{1, \ldots, n\}$, we have proved that $x(s+1)=\mathbb{e}_{i}$ in Lemma 1 . If instead $x(s)$ is not a vertex of the simplex, then $x_{i}(s)<1$ for all $i$ so that $\alpha(s) \neq 0$ and, therefore, $x_{i}(s+1)=\frac{\alpha(s) c_{i}}{1-x_{i}(s)}$, or equivalently

$$
x(s+1)=\alpha(s)\left(\frac{c_{1}}{1-x_{1}(s)}, \ldots, \frac{c_{n}}{1-x_{n}(s)}\right) .
$$

That is to say, $x(s+1)=F(x(s))$ as claimed.

Here it is useful to look at two example relative interaction matrices and corresponding dynamics. First, if the relative interaction matrix $C$ is not only row-stochastic, irreducible and with zero diagonal, but also doubly stochastic, then its dominant left eigenvector is $\mathbb{1}_{n} / n$ and so the DeGrootFriedkin map simplifies to

$F(x)= \begin{cases}\mathbb{e}_{i}, & \text { if } x=\mathbb{e}_{i} \text { for any } i \in\{1, \ldots, n\}, \\ \left(\frac{1}{1-x_{1}}, \ldots, \frac{1}{1-x_{n}}\right) / \sum_{i=1}^{n} \frac{1}{1-x_{i}}, & \text { otherwise }\end{cases}$

Second, note that, if $n=2$, then $C$ is always doubly-stochastic and, for any $\left(x_{1}, x_{2}\right) \in \Delta_{2}$ with strictly positive components, $F$ satisfies $F\left(x_{1}, x_{2}\right)=\left(x_{1}, x_{2}\right)$.

\section{F. Centrality measures}

As Lemma 3 characterizes, the dominant left eigenvector $c$ of the relative interaction matrix $C$ (assumed irreducible) plays a key role in the dynamics analysis of DeGroot-Friedkin model. Specifically, the relative interaction matrix $C$ plays no direct role and the only parameters appearing in the DeGrootFriedkin dynamic model is $c \in \Delta_{n}$.

In the language of (Bonacich, 1972), the entries of $c$ are the eigenvector centrality scores for the weighted digraph with adjacency matrix $C^{T}$. (In our setup, if one regards our rowstochastic matrix $C$ as an adjacency matrix, then its dominant right eigenvector $\mathbb{1}_{n}$ is not informative and it is precisely the left dominant left eigenvector $c$ that measures the influence of a node on all others.) In what follows, we refer to $c_{i}$ as the eigenvector centrality score of node $i$ (with respect to the adjacency matrix $C^{T}$ ) and we refer to the node with the largest entry of $c$, if it exists unique, as the eigenvector center.

Remark 4 As proposed in (Friedkin, 1991), the total effect centrality of node $i$ in an opinion formation process (described by an interpersonal influence matrix and potentially other 
parameters) is the average effect of the opinion of node $i$ on the final opinions at all other nodes. With this nomenclature, the vector $c$ (the dominant left eigenvector of the relative interaction matrix $C$ ) is therefore the total effect centrality vector for the matrix $C$ regarded as the influence matrix in a DeGroot opinion formation process.

\section{EQUILIBRIA AND CONVERGENCE ANALYSIS FOR DOUBLY-STOCHASTIC RELATIVE INTERACTIONS}

Theorem 5 (DeGroot-Friedkin model with doublystochastic relative interactions) For $n \geq 3$, consider the DeGroot-Friedkin dynamical system $x(s+1)=F(x(s))$ defined by a relative interaction matrix $C \in \mathbb{R}^{n \times n}$ that is doubly stochastic, irreducible and with zero diagonal. The following statements hold true:

(i) the fixed points of $F$ are the vertices $\left\{\mathbb{e}_{1}, \ldots, \mathbb{e}_{n}\right\}$ and the equal-weight vector $\mathbb{1}_{n} / n$,

(ii) for any initial condition $x(1) \in \Delta_{n} \backslash\left\{\mathbb{e}_{1}, \ldots, \mathbb{e}_{n}\right\}$, the corresponding solution $\{x(s)\}_{s \in \mathbb{N}}$ has the following properties:

a) if $x_{i}(1)>x_{j}(1)$ for some $i \neq j$, then $x_{i}(s)>$ $x_{j}(s)$ for all future issues $s \in \mathbb{N}$,

b) if $x(1) \neq \mathbb{1}_{n} / n$, then the function $s \mapsto$ $\max \left\{x_{1}(s), \ldots, x_{n}(s)\right\} / \min \left\{x_{1}(s), \ldots, x_{n}(s)\right\}$, for $s \geq 2$, is bounded and strictly decreasing, and c) $\lim _{s \rightarrow \infty} x(s)=\mathbb{1}_{n} / n$.

(iii) for any initial condition $x(1) \in \Delta_{n} \backslash\left\{\mathbb{e}_{1}, \ldots, \mathbb{e}_{n}\right\}$, the dominant left eigenvector of the influence matrix $W(x(s))$ satisfies $\lim _{s \rightarrow \infty} w(x(s))=\mathbb{1}_{n} / n$.

Statement (ii.c) has the following implication: all solutions starting away from a leader vector converge asymptotically to the equal-weight vector. The case of doubly-stochastic DeGroot models was first characterized in (Harary, 1959, Theorem 14).

Sketch proof of Theorem 5: Fact (i) is straightforward.

Regarding fact (ii), define the scalar function $f:[0,1[\rightarrow$ $[1, \infty[$ by $f(x)=x /(1-x)$ and note that $F(x)=$ $\alpha\left(f\left(x_{1}\right), \ldots, f\left(x_{n}\right)\right)^{T}$ for the appropriate $\alpha>0$ defined above. It is immediate to see that $f$ is a strictly increasing function. Therefore, $x_{i}>x_{j}$ implies $f\left(x_{i}\right)>f\left(x_{j}\right)$ and, in turn, $\alpha f\left(x_{i}\right)>\alpha f\left(x_{j}\right)$. This proves the first property in fact (ii).

We now perform a Lyapunov analysis to establish convergence. For the discrete-time dynamical system $x(s+1)=$ $F(x(s))$ with $F$ defined in equation (3), we define the continuous Lyapunov function candidate $V: \operatorname{interior}\left(\Delta_{n}\right) \rightarrow \mathbb{R}$ by

$$
V(x)=\frac{\max \left\{x_{1}, \ldots, x_{n}\right\}}{\min \left\{x_{1}, \ldots, x_{n}\right\}} .
$$

Here, the set interior $\left(\Delta_{n}\right)=\left\{x \in \mathbb{R}^{n} \mid x>0, \mathbb{1}_{n}^{T} x=\right.$ $1\}$ is the interior of the simplex $\Delta_{n}$. The property $x \in$ interior $\left(\Delta_{n}\right) \Longrightarrow \min \left\{x_{1}, \ldots, x_{n}\right\}>0$ implies that $V$ is well defined. Moreover, note that interior $\left(\Delta_{n}\right)$ is invariant for $F$, because $0<x_{i}<1$ implies $f\left(x_{i}\right)>0$, so that every trajectory starting in interior $\left(\Delta_{n}\right)$ remains in it for ever.
Next, given $x \in \operatorname{interior}\left(\Delta_{n}\right)$ and given the shorthands $x_{\max }=\max \left\{x_{1}, \ldots, x_{n}\right\}>0$ and $x_{\min }=$ $\min \left\{x_{1}, \ldots, x_{n}\right\}>0$, it can be shown that

$$
x_{\max }>x_{\min } \Longrightarrow \frac{f\left(x_{\max }\right)}{f\left(x_{\min }\right)}<\frac{x_{\max }}{x_{\min }} .
$$

This inequality implies that, for all $x \in \operatorname{interior}\left(\Delta_{n}\right) \backslash\left\{\mathbb{1}_{n} / n\right\}$,

$$
V(F(x))<V(x) .
$$

In summary, 1) any sublevel set of $V$ is compact and invariant, 2) $V$ is strictly decreasing anywhere on interior $\left(\Delta_{n}\right) \backslash\left\{\mathbb{1}_{n} / n\right\}$, 3 ) both $F$ and $V$ are continuous. Under these assumption, the Lyapunov theorem for discrete-time dynamical systems implies that every trajectory starting in interior $\left(\Delta_{n}\right)$ converges asymptotically to the equilibrium point $\mathbb{1}_{n} / n$.

To complete the proof of the second property in (ii), consider a point $\bar{x}$ on the boundary of $\Delta_{n}$ but distinct from the vertices $\left\{\mathbb{e}_{1}, \ldots, \mathbb{e}_{n}\right\}$. It is easy to see that $F(\bar{x}) \in \operatorname{interior}\left(\Delta_{n}\right)$. Therefore, every trajectory starting in $\Delta_{n} \backslash\left\{\mathbb{e}_{1}, \ldots, \mathbb{e}_{n}\right\}$ converges asymptotically to $\mathbb{1}_{n} / n$.

Regarding fact (iii), given $\lim _{s \rightarrow \infty} x(s)=\mathbb{1}_{n} / n$ and given the definition of $W(x)$ in equation (2),

$$
\begin{array}{r}
\lim _{s \rightarrow \infty} W(x(s))=\lim _{s \rightarrow \infty}\left(\operatorname{diag}(x(s)) I_{n}+\operatorname{diag}\left(\mathbb{1}_{n}-x(s)\right) C\right) \\
=I_{n} / n+(n-1) C / n .
\end{array}
$$

Because $C$ is doubly-stochastic, we know $\mathbb{1}_{n}^{T} C=\mathbb{1}_{n}^{T}$ and compute

$$
\frac{\mathbb{1}_{n}^{T}}{n}\left(\lim _{s \rightarrow \infty} W(x(s))\right)=\frac{\mathbb{1}_{n}^{T}}{n}\left(I_{n} / n+(n-1) C / n\right)=\frac{\mathbb{1}_{n}^{T}}{n} .
$$

Moreover, since $x \mapsto W(x)$ is an analytic function and 1 is a simple eigenvalue of $W(x)$ for any $x \in \Delta_{n}$, the dominant left eigenvector of $W(x)$ is known to be an analytic function of $x$ near $\mathbb{1}_{n}^{T} / n$; e.g., see (Lax, 1997). Hence, $\lim _{s \rightarrow \infty} w(x(s))=$ $\mathbb{1}_{n} / n$.

We conclude this section with some example simulations in dimension $n=3$. Trajectories of the DeGroot-Friedkin dynamics with a doubly-stochastic $C$ are depicted in Fig. 1. As predicted, all trajectories converge to the equal-weight configuration $\mathbb{1}_{3} / 3$.

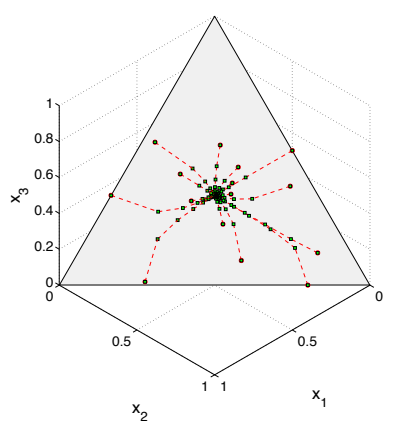

Fig. 1. DeGroot-Friedkin dynamics with a doubly-stochastic $C$ : starting from several sample initial states in $\Delta_{3} \backslash\left\{\mathbb{e}_{1}, \mathbb{e}_{2}, \mathbb{e}_{3}\right\}$, all corresponding state trajectories converge to $\mathbb{1}_{3} / 3$. 


\section{EQUILIBRIA AND CONVERGENCE ANALYSIS FOR RELATIVE INTERACTIONS WITH STAR TOPOLOGY}

In this section we assume the digraph $G(C)$ associated to the relative interaction matrix $C$ has star topology, that is, (i) there is a node, called the center node, which has directed edges to and from all other nodes and (ii) every other node, called a leaf, has a directed edge to and from only the center node. For illustration purposes, consider the star digraph in Fig. 2 with corresponding row-stochastic adjacency matrix

$$
C=\left[\begin{array}{ccc}
0 & 1 & 0 \\
1 / 2 & 0 & 1 / 2 \\
0 & 1 & 0
\end{array}\right]
$$

Because the properties that $G(C)$ has star topology and that $n=2$ together imply that the matrix $C$ is doubly-stochastic and because we already analyzed that case, we assume $n \geq 3$ in what follows.

Lemma 6 (The dominant left eigenvector of a star digraph) For $n \geq 3$ and $C$ row stochastic, irreducible with zero diagonal, the dominant left eigenvector $c \in \Delta_{n}$ of $C$ satisfies $\max \left\{c_{1}, \ldots, c_{n}\right\} \leq 0.5$. Moreover, the following statements are equivalent:

(i) the node $i$ satisfies $c_{i}=0.5>c_{j}$, for all $j \in$ $\{1, \ldots, n\} \backslash\{i\}$, so that $i$ is the eigenvector center,

(ii) the digraph $G(C)$ has star topology with center node $i$, that is, $C_{j i}=1$ for all $j \in\{1, \ldots, n\} \backslash\{i\}$.

Theorem 7 (DeGroot-Friedkin model with row-stochastic relative interactions and star network topology) For $n \geq 3$, consider the DeGroot-Friedkin dynamical system $x(s+1)=$ $F(x(s))$ defined by a relative interaction matrix $C \in \mathbb{R}^{n \times n}$ that is row stochastic, irreducible, with zero diagonal, with star topology and with center node 1 . The following statements hold true:

(i) the fixed points of $F$ are the vertices $\left\{\mathbb{e}_{1}, \ldots, \mathbb{e}_{n}\right\}$,

(ii) for any initial condition $x(1) \in \Delta_{n} \backslash\left\{\mathbb{e}_{1}, \ldots, \mathbb{e}_{n}\right\}$, the corresponding solution $\{x(s)\}_{s \in \mathbb{N}}$ has the following properties:

a) the function $s \mapsto 1-x_{1}(s), s \in \mathbb{N}$, is bounded and strictly decreasing, and

b) $\lim _{s \rightarrow \infty} x(s)=\mathbb{e}_{1}$.

(iii) for any initial condition $x(1) \in \Delta_{n} \backslash\left\{\mathbb{e}_{1}, \ldots, \mathbb{e}_{n}\right\}$, the dominant left eigenvector of the influence matrix $W(x(s))$ satisfies $\lim _{s \rightarrow \infty} w(x(s))=\mathbb{e}_{1}$.

Sketch of proof: Fact (i) is straightforward: there does not exist an equilibrium in $\Delta_{n} \backslash\left\{\mathbb{e}_{1}, \ldots, \mathbb{e}_{n}\right\}$ as $c_{1}=0.5$.

Regarding fact (ii), consider an initial state $x \in \Delta_{n}$ । $\left\{\mathbb{e}_{1}, \ldots, \mathbb{e}_{n}\right\}$. As $x_{1} \neq 1, x_{1}-F_{1}(x)=x_{1}-\frac{\alpha(x) c_{1}}{1-x_{1}}$, where $\alpha(x)=\frac{1}{\frac{c_{1}}{1-x_{1}}+\sum_{j=2}^{n} \frac{c_{j}}{1-x_{j}}}$. If $x_{1}=0$, then $F_{1}(x)=\alpha(x) c_{1}>$ 0 , and hence $x_{1}-F_{1}(x)<0$. If $x_{1}>0$, we can show that $c_{1}=0.5$ implies $\sum_{j=2}^{n} \frac{c_{j}}{1-x_{j}}<\frac{c_{1}}{x_{1}}$, which implies

$$
\sum_{j=2}^{n} \frac{c_{j}}{1-x_{j}}+\frac{c_{1}}{1-x_{1}}<\frac{c_{1}}{x_{1}}+\frac{c_{1}}{1-x_{1}}=\frac{c_{1}}{\left(1-x_{1}\right) x_{1}}
$$

which is equivalent to $\alpha(x)>\left(1-x_{1}\right) x_{1} / c_{1}$. Hence, $x_{1}-$ $F_{1}(x)=\frac{c_{1}}{1-x_{1}}\left(\frac{\left(1-x_{1}\right) x_{1}}{c_{1}}-\alpha(x)\right)<0$ for all $x_{1} \neq 1$ and $x_{1}-F_{1}(x)=0$ for $x_{1}=1$ by definition.

Define a Lyapunov function candidate $V(x)=1-x_{1}$. It is clear that 1) any sublevel set of $V$ is compact and invariant, 2) $V$ is strictly decreasing anywhere in $\left.\Delta_{n} \backslash\left\{\mathbb{e}_{1}, \ldots, \mathbb{e}_{n}\right\}, 3\right) V$ and $F$ are continuous. Therefore, every trajectory starting in $\Delta_{n} \backslash\left\{\mathbb{e}_{1}, \ldots, \mathbb{e}_{n}\right\}$ converges asymptotically to the equilibrium point $\mathbb{e}_{1}$ by the Lyapunov theorem for discrete-time dynamical system.

Regarding fact (iii), given $\lim _{s \rightarrow \infty} x(s)=\mathbb{e}_{1}$, similarly as discussed in the proof of Theorem 5 (iii), we can show $\lim _{s \rightarrow \infty} w(x(s))=\mathbb{e}_{1}$.

For the relative interaction matrix in equation (6), $\left\{\mathbb{e}_{1}, \mathbb{e}_{2}, \mathbb{e}_{3}\right\}$ are the only equilibria as predicted by Theorem 7 . The theorem also establishes that all trajectories converge to $\mathbb{e}_{2}$ if the initial states are not these vertices; this is indeed illustrated by Fig. 3 .
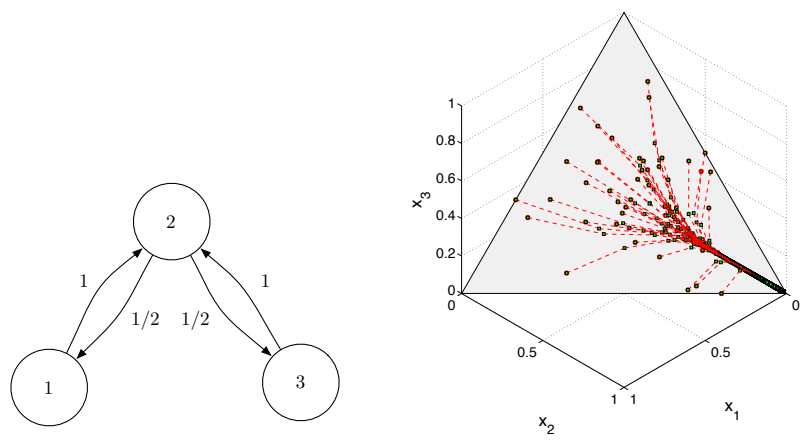

Fig. 2. Star topology with cen- Fig. 3. DeGroot-Friedkin dynamics with ter node 2: the dominant left star topology as shown in Fig. 2 and eigenvector of $C$ in (6) is $c=$ a row-stochastic $C$ as given in equa$(1 / 4,1 / 2,1 / 4)$ and the center tion (6): starting from several sample node is also the eigenvector center initial states in $\Delta_{3} \backslash\left\{\mathbb{e}_{1}, \mathbb{e}_{2}, \mathbb{e}_{3}\right\}$, all (i.e., the node with the largest entry corresponding state trajectories converge in the dominant left eigenvector). to the vertex $\mathbb{e}_{2}$

\section{Mathematical AND STATistical ANALYSis OF THE GENERAL CASE}

In general circumstances, the relative interaction matrix $C$ is only row-stochastic and neither doubly stochastic nor representing a star topology. We present some preliminary results about this general case and refer the reader to forthcoming publications for their proofs.

Theorem 8 (DeGroot-Friedkin model with row-stochastic relative interactions) For $n \geq 3$, consider the DeGrootFriedkin dynamical system $x(s+1)=F(x(s))$ defined by a relative interaction matrix $C \in \mathbb{R}^{n \times n}$ that is row stochastic, irreducible and with zero diagonal. Assume $G(C)$ does not have star topology and let $c$ be the dominant left eigenvector of $C$. The following statements hold true:

(i) the vertices $\left\{\mathbb{e}_{1}, \ldots, \mathbb{e}_{n}\right\}$ are fixed points of $F$ and no other point on the boundary of $\Delta_{n}$ is a fixed point,

(ii) every equilibrium $\mathbb{e}_{1}, \ldots, \mathbb{e}_{n}$ is unstable, 
(iii) if there exists a fixed point $x^{*} \in \Delta_{n} \backslash\left\{\mathbb{e}_{1}, \ldots, \mathbb{e}_{n}\right\}$ of $F$, then

a) $x^{*}$ is the unique fixed point in $\Delta_{n} \backslash\left\{\mathbb{e}_{1}, \ldots, \mathbb{e}_{n}\right\}$,

b) if $c_{i}>c_{j}$, then $x_{i}^{*}>x_{j}^{*}$, and

c) if $c_{i}=c_{j}$, then $x_{i}^{*}=x_{j}^{*}$.

An example application to the Krackhardt's advice network

Krackhardt (1987) presents data about an advice network (as shown in Fig. 4) in a manufacturing organization on the west coast of the United States. The organization has 21 managers and the directed advice network $C$ is formulated based upon who sought advice from whom. If agent $i$ asks for advice from $m$ different agents, then we assume, as done for example by Jackson (2010), that $C_{i j}=1 / m$ for $j$ in these $m$ agents, and $C_{i k}=0$ for any other agent $k$. Moreover, self-weighting is not considered in $C$, that is, $C_{i i}=0$ for all $i \in\{1, \ldots, 21\}$. Such a matrix $C$ has a unique dominant left eigenvector

$$
\begin{aligned}
c=\left[\begin{array}{lllllll}
0.0470 & 0.1320 & 0.0388 & 0.0516 & 0.0022 & 0 & 0.1434 \ldots \\
0.0074 & 0.0143 & 0.0237 & 0.0528 & 0.0512 & 0 & 0.0716 \ldots \\
0.0143 & 0 & 0 & 0.1012 & 0.0065 & 0.0366 & 0.2053
\end{array}\right]^{T} .
\end{aligned}
$$

We simulate the DeGroot-Friedkin model on this Krackhardt's advice network with 27000 randomly chosen initial states $x(1) \in \Delta_{21}$. The simulations show that all dynamical trajectories converge to a unique equilibrium self-weight vector $x^{*}$, given by

$$
\begin{aligned}
x^{*}=\left[\begin{array}{llllllll}
0.0432 & 0.1339 & 0.0354 & 0.0476 & 0.0020 & 0 & 0.1478 \ldots \\
0.0065 & 0.0128 & 0.0212 & 0.0487 & 0.0472 & 0 & 0.0674 \ldots \\
0.0127 & 0 & 0 & 0.0987 & 0.0058 & 0.0332 & 0.2360
\end{array}\right]^{T} .
\end{aligned}
$$

By probability estimation via Monte Carlo methods, our simulations establish the following statement: With $99 \%$ confidence level, there is at least $99 \%$ probability that any self-weight trajectories $s \mapsto x(s)$ converges to the equilibrium $x^{*}$ in Krackhardt's advice network.

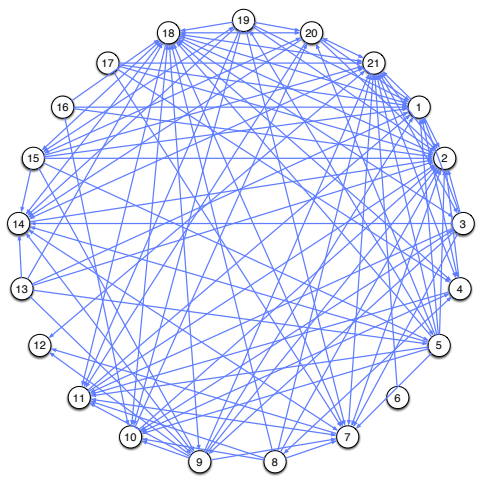

Fig. 4. Krackhardt's advice network.

\section{FUTURE WORK}

Much work remains to be done in order to understand the robustness of these results to our choice of opinion formation process. Indeed, qualitatively different results are obtained in the (Friedkin, 2011) model. This model allows for stubborn individuals who are resistant to altering whatever opinion they happen to hold at a particular time and couples such stubbornness with an ongoing attachment to initial opinions. The result of allowing ongoing attachments to initial opinions is an influence network evolution that presents an equilibrium concentration of total relative control on one or two members. We do not as yet understand the mathematical foundations of this (Friedkin, 2011) simulation result, which we have replicated.

\section{REFERENCES}

D. Acemoglu, G. Como, F. Fagnani, and A. Ozdaglar. Opinion fluctuations and disagreement in social networks. Mathematics of Operation Research, 38(1):1-27, 2013.

V. D. Blondel, J. M. Hendrickx, and J. N. Tsitsiklis. On Krause's multi-agent consensus model with state-dependent connectivity. IEEE Transactions on Automatic Control, 54 (11):2586-2597, 2009.

P. Bonacich. Technique for analyzing overlapping memberships. Sociological Methodology, 4:176-185, 1972.

C. H. Cooley. Human Nature and the Social Order. Scribner's, New York, 1902.

G. Deffuant, D. Neau, F. Amblard, and G. Weisbuch. Mixing beliefs among interacting agents. Advances in Complex Systems, 3(1/4):87-98, 2000.

M. H. DeGroot. Reaching a consensus. Journal of the American Statistical Association, 69(345):118-121, 1974.

J. R. P. French. A formal theory of social power. Psychological Review, 63(3):181-194, 1956.

N. E. Friedkin. A formal theory of reflected appraisals in the evolution of power. Administrative Science Quarterly, 56 (4):501-529, 2011.

N. E. Friedkin. Theoretical foundations for centrality measures. American Journal of Sociology, 96(6):1478-1504, 1991.

N. E. Friedkin and E. C. Johnsen. Social influence networks and opinion change. In E. J. Lawler and M. W. Macy, editors, Advances in Group Processes, volume 16, pages 1-29. JAI Press, 1999.

V. Gecas and M. L. Schwalbe. Beyond the looking-glass self: Social structure and efficacy-based self-esteem. Social Psychology Quarterly, 46(2):77-88, 1983.

F. Harary. A criterion for unanimity in French's theory of social power. In D. Cartwright, editor, Studies in Social Power, pages 168-182. University of Michigan, 1959.

R. Hegselmann and U. Krause. Opinion dynamics and bounded confidence models, analysis, and simulations. Journal of Artificial Societies and Social Simulation, 5(3), 2002.

M. O. Jackson. Social and Economic Networks. Princeton University Press, 2010.

D. Krackhardt. Cognitive social structures. Social Networks, 9(2):109-134, 1987.

P. D. Lax. Linear Algebra. Pure and Applied Mathematics. Wiley, 1997. 